Tôhoku Math. Journ.

Vol. 18, No. 4, 1966

\title{
ON A CLASS OF OPERATORS
}

\author{
VASIle IstrăȚescu, TeIshirô SAITÔ AND TAKAShi Yoshino
}

(Received September 20, 1966)

1. We consider bounded linear operators on a Hilbert space $H$. Denote by $\sigma(T)$ the spectrum, by $\sigma_{p}(T)$ the point spectrum and by $\pi(T)$ the approximate point spectrum of an operator $T$. As in [3], an operator $T$ is said to be of class $(N)$ in case $\left\|T^{2} x\right\| \geqq\|T x\|^{2}$ for all unit vectors $x \in H$. A. Wintner [8] calls an operator $T$ normaloid if $\|T\|=\sup \{|(T x, x)|: x \in H,\|x\|=1\}$. It is known that $T$ is normaloid if and only if $\|T\|=\sup \{|\lambda|: \lambda \in \sigma(T)\}$ or equivalently, $\left\|T^{n}\right\|=\|T\|^{n}$ for $n=1,2, \cdots$. If $T$ is a hyponormal operator, that is $\|T x\| \geqq\left\|T^{*} x\right\|$ for all $x \in H$, then $T$ is of class $(N)$. In fact, if $T$ is a hyponormal operator, we have

$$
\|T x\|^{2}=\left(T^{*} T x, x\right) \leqq\left\|T^{*}(T x)\right\| \leqq\left\|T^{2} x\right\|,
$$

for any unit vector $x \in H$.

2. In this section we prove some theorems on an operator of class $(N)$. The following theorem is suggested by [6] and [7].

THEOREM 1. For an operator $T$ of class $(N)$,

(i) $T$ is normaloid,

and

(ii) $T^{-1}$ is also of class $(N)$ if $T$ is invertible.

ProOF. To prove (i), it is sufficient to show that $\left\|T^{n} x\right\| \geqq\|T x\|^{n}$ for each unit vector $x \in H$ and $n=1,2, \cdots$. If $n \leqq 2$, the inequality is obvious by the definition of class $(N)$. Suppose that $\left\|T^{k} x\right\| \geqq\|T x\|^{k}$ for $k=1,2, \cdots, n$ and $x \in H,\|x\|=1$. Then

$$
\begin{aligned}
\left\|T^{n+1} x\right\| & =\|T x\|\left\|T^{n} \frac{T x}{\|T x\|}\right\| \geqq\|T x\|\left\|T \frac{T x}{\|T x\|}\right\|^{n} \\
& =\|T x\|^{1-n}\left\|T^{2} x\right\|^{n} \geqq\|T x\|^{1-n}\|T x\|^{2 n}=\|T x\|^{n+1}
\end{aligned}
$$


for $x \in H,\|x\|=1$ and the induction is completed.

To prove (ii), let $y \in H$ be an arbitrary unit vector. Then there is an $x \in H$ such as $y=T^{2} x$. As $T$ is of class $(N)$, we have

$$
\begin{aligned}
\left\|T^{-1} y\right\|^{2} & =\|T x\|^{2}=\|x\|^{2}\left\|T \frac{x}{\|x\|}\right\|^{2} \leqq\|x\|^{2}\left\|T^{2} \frac{x}{\|x\|}\right\| \\
& =\|x\|\left\|T^{2} x\right\|=\|x\|\|y\|=\|x\|=\left\|T^{-2} y\right\| .
\end{aligned}
$$

and $T^{-1}$ is of class $(N)$.

As an immediate consequence of Theorem 1 we have the following corollary.

COROLLARY. If $T$ is an operator of class $(N)$ and $\sigma(T)$ lies on the unit circle, $T$ is a unitary operator.

In the case of hyponormal operator this is nothing but a reshlt of [6] and [7].

PROOF. If $\sigma(T)$ lies on the unit circle, then $\|T\|=\left\|T^{-1}\right\|=1$ by Theorem 1 . Hence we have

$$
\begin{aligned}
\|x\| \geqq\|T x\| & =\left\|T^{-1} x\right\|\left\|T^{2} \frac{T^{-1} x}{\left\|T^{-1} x\right\|}\right\| \geqq\left\|T^{-1} x\right\|\left\|T \frac{T^{-1} x}{\left\|T^{-1} x\right\|}\right\|^{2} \\
& =\frac{\|x\|^{2}}{\left\|T^{-1} x\right\|} \geqq\|x\|,
\end{aligned}
$$

and $\|T x\|=\|x\|$ for $x \in H$ and $T$ is a unitary operator.

In [1] T. Andô has proved that every completely continuous hyponormal operator is necessarily normal. The following theorem is a slight generalization of it.

THEOREM 2. Let $T$ be an operator of class $(N)$ such that $T^{* p_{1}} T^{q_{1}} \ldots$ $T^{* p_{m}} T^{q_{m}}$ is completely continuous for some non-negative integers $p_{1}, q_{1}, \cdots$, $p_{m}, q_{m}$. Then $T$ is necessarily a normal operator.

To prove the theorem, we shall need some preliminary lemmas. The following lemma is well-known (see [5]), but we cite here for convenience.

LEMMA 1. For any operator $T, \sigma(T) \cap\{\lambda:|\lambda|=\|T\|\} \subset \pi(T)$, and if $\mu \in \sigma(T) \cap\{\lambda:|\lambda|=\|T\|\}, T x_{n}-\mu x_{n} \rightarrow 0(n \rightarrow \infty)$ is equivalent to $T^{*} x_{n}-\bar{\mu} x_{n}$ $\rightarrow 0(n \rightarrow \infty)$ for any sequence $\left\{x_{n}\right\}$ of unit vectors in $H$. 
The essential part of our proof is the following lemma.

LEMMA 2. Let $T$ be an operator such that $T^{* p_{1}} T^{q_{1}} \cdots T^{* p_{m}} T^{q_{m}}$ is completety continuous for some non-negative integers $p_{1}, q_{1}, \cdots, p_{m}, q_{m}$. Then the condition $\mu \in \sigma\left(T^{\prime}\right) \cap\{\lambda:|\lambda|=\|T\|\}$ implies $\mu \in \sigma_{p}(T)$ and $\bar{\mu} \in \sigma_{p}\left(T^{*}\right)$.

PROOF. To simplify the notations, we shall treat the case where $T^{*}{ }^{q} T^{q}$ is completely continuous for some non-negative integers $p$ and $q$. Since $\mu \in \sigma(T) \cap\{\lambda:|\lambda|=\|T\|\}$, there is a sequence $\left\{x_{n}\right\}$ of unit vectors in $H$ such as $\left\|T x_{n}-\mu x_{n}\right\| \rightarrow 0$ and $\left\|T^{*} p T^{q} x_{n}-\bar{\mu}^{p} \mu^{q} x_{n}\right\|_{1} \rightarrow 0(n \rightarrow \infty)$ by Lemma 1 . As $T^{*} p T^{q}$ is completely continuous, we may assume that (if necessary, by choosing a suitable subsequence) the sequence $\left\{T^{*}{ }^{p} T^{q} x_{n}\right\}$ converges to a certain vector $x \in H$. Let $x_{0}$ be $x / \bar{\mu}^{p} \mu^{q}$, then $\left\|x_{n}-x_{0}\right\| \rightarrow 0(n \rightarrow \infty)$. Therefore $T x_{0}=\mu x_{0}$ and so $T^{*} x_{0}=\bar{\mu} x_{0}$ by Lemma 1, i.e., $\mu \in \sigma_{p}(T)$ and $\bar{\mu} \in \sigma_{p}\left(T^{*}\right)$.

PRoOF OF THEOREM 2. Throughout the proof, $\Re_{T}(\lambda)$ means the $\lambda$-th proper subspace of an operator $T$, that is $\Re_{T}(\lambda)=\{x \in H: T x=\lambda x\}$. At first, we notice that there is at least one $\lambda \in \sigma_{p}(T)$ such as $\Re_{T}(\lambda) \cap \Re_{T^{*}}(\bar{\lambda}) \neq(0)$. In fact, since $T$ is normaloid by Theorem 1 , there is a $\lambda_{0} \in \sigma \cdot(T)$ such as $\left|\lambda_{0}\right|$ $=\|T\|$. Thus $\lambda_{0} \in \sigma_{p}(T)$ and $\bar{\lambda}_{0} \in \sigma_{p}\left(T^{*}\right)$ by Lemma 2 and $\Re_{T}\left(\lambda_{0}\right) \cap \Re_{T^{*}}\left(\bar{\lambda}_{0}\right) \neq(0)$ by the proof of Lemma 2. Now it is easy to see that $\left\{\Re_{T}(\lambda) \cap \Re_{T^{*}}(\lambda): \lambda \in \sigma_{p}(T)\right\}$ is a mutually orthogonal family. Let $H_{0}$ be $\sum_{\lambda \in \sigma_{p}(T)} \oplus\left(\Re_{T}(\lambda) \cap \mathfrak{X}_{T^{*}}(\bar{\lambda})\right)$, then $H_{0}$ reduces $T$ and the restriction of $T$ onto $H_{0}$ is normal. To complete the proof of the theorem, we have only to prove that the restriction $T_{1}$ of $T$ onto $H_{1}=H_{0}^{\perp}$ is 0 . Suppose the contrary. Then $T_{1}$ is a non-zero operator of class $(N)$ and $T_{1}^{* p} T_{1}^{q}$ is also completely continuous. By Theorem $1, T_{1}$ is normaloid and there exists a $\mu \in \sigma\left(T_{1}\right)$ such as $|\mu|=\left\|T_{1}\right\|$. Hence $T_{1} x=\mu x$ for some non-zero vector $x \in H_{1}$ and then $T_{1}^{*} x=\bar{\mu} x$ by the proof of Lemma 2. Therefore $\Re_{T}(\mu) \cap \Re_{T^{*}}(\bar{\mu}) \neq(0)$ and this is orthogonal to $H_{0}$. This is a contradiction.

\section{REFERENCES}

[1] T. ANDô, On hyponormal operators, Proc. Amer. Math. Soc., 14(1963), 290-291.

[2] S. K. Berberian, Introduction to Hilbert space, Oxford Univ. Press, New York, 1961.

[3] V. Istratescu, On some hyponormal operators, to appear in Pacific Journ. Math.

[4] G. H. Orland, On a class of operators, Proc. Amer. Math. Soc., 15(1964), 75-79.

[5] M. SchreIBER, On the spectrum of a contraction, Proc. Amer. Math. Soc., 12(1961), 709-713.

[6] J. G. STAMPfli, Hyponormal operators and spectral density, Trans. Amer. Math. Soc., $117(1965)$, 469-476. 
[7] T. Yoshino, On the spectrum of a hyponormal operator, Tôhoku Math. Journ., 17 (1965), 305-309.

[8] A. WintneR, Zur Theorie der beschrankten Bilineärformen, Math. Zeit., 30(1929), 228-282.

INSTITUTE OF MATHEMATICS, RUMANIAN ACADEMY, AND

TÔHOKU UNIVERSITY. 